Gut and Liver, Vol. 9, No. 5, September 2015, pp. 641-648

\title{
The Usefulness of a Novel Screening Kit for Colorectal Cancer Using the Immunochromatographic Fecal Tumor M2 Pyruvate Kinase Test
}

Yong Cheol Kim*, Jeong Ho Kim ${ }^{\dagger}$, Dae Young Cheung*, Tae Ho Kim*, Eun Jung Jun,* Jung-Whan Oh*, Chang Whan Kim*, Woo Chul Chung*, Byung-Wook Kim*, Sung Soo Kim*, Jin II Kim*, Soo-Heon Park*, and Jae Kwang Kim*

*Department of Internal Medicine, The Catholic University of Korea College of Medicine, Seoul, and ${ }^{\dagger}$ Division of Gastroenterology, Department of Internal Medicine, Myongiji Hospital, Seonam University College of Medicine, Goyang, Korea

Background/Aims: M2 pyruvate kinase (M2-PK) is an enzyme that is produced in undifferentiated and proliferating tissues. This study aims to evaluate the usefulness of the immunochromatographic M2 pyruvate kinase (iM2-PK) for the screening of colorectal cancer (CRC) and premalignant lesions. Methods: Healthy volunteers and patients with colorectal neoplasia were enrolled in six academic hospitals in the capital province of Korea. The iM2-PK value was compared with the immunochromatographic fecal occult blood test (iFOBT) and fecal tumor M2-PK enzyme-linked immunosorbent assay (ELISA). Results: A total of 323 subjects were enrolled. The sensitivity of iM2-PK for CRC was $92.8 \%$, which was superior to iFOBT (47.5\%, p<0.0001). For adenomatous lesions, the sensitivity of iM2-PK was $69.4 \%$, which was also superior to iFOBT (12.1\%, p<0.001). Compared with M2-PK ELISA, iM2-PK exhibited significantly enhanced sensitivity for CRC (97.5\% vs $80.0 \%, p=0.0289$ ). The sensitivity of iM2$\mathrm{PK}$ was higher in advanced stages of CRC compared with cancers confined to the mucosa and submucosa $(p<0.05)$. However, lymph node metastasis had no influence on the sensitivity of iM2-PK. Conclusions: The iM2-PK exhibited increased sensitivity for identifying $\mathrm{CRC}$ and adenomatous lesions compared with iFOBT. Given its rapid results and convenience, CRC screening using iM2-PK is promising. (Gut Liver 2015;9:641-648)

Key Words: Colorectal neoplasms; Fecal occult blood test; Mass screening; Tumor M2 pyruvate kinase

\section{INTRODUCTION}

Colorectal cancer (CRC) is the second most common cancer in males and the third in females in Korea. A total of 25,782 people were diagnosed with CRC in 2010 and the average annual increasing rate of diagnosing CRC was about 6.7\% per year. One of the reasons for this upward trend in diagnosing CRC may be related to the increase in screening tests such as fecal occult blood test (FOBT) by national health insurance system and the rise in the number of colonoscopy by many private health promotion clinics. ${ }^{1}$ In global statistics, CRC is the third most commonly diagnosed cancer in males and the second in females with over 1.2 million new cancer cases and 608,700 deaths occurred in 2008. ${ }^{2}$

Colonoscopy is a gold standard method for diagnosing CRC. However, the acceptance of colonoscopy is quite low because of its high cost and invasiveness. FOBT has been widely performed in screening programs for diagnosing CRC around the world and has proven a certain benefit. According to a previous metaanalysis, the sensitivity, specificity and positive predictive value of immunochromatographic fecal occult blood test (iFOBT) in CRC were 67\%, 85\%, and 41\%, respectively. ${ }^{3}$ Study for genetic alterations (such as K-ras and p53) is a very interesting approach, but these tests are expensive and unavailable in daily practice. ${ }^{4,5}$ And it is bothersome to get fresh stool samples for DNA isolation.

The M2 pyruvate kinase (M2-PK) is an isoenzyme of pyruvate kinase mainly produced in undifferentiated and proliferating tissues. In cancer cells, M2-PK regulates the synthetic balance between adenosine triphosphate and macromolecules. It plays

Correspondence to: Dae Young Cheung ${ }^{1}$ and Jae Kwang Kim ${ }^{2}$

${ }^{1}$ Department of Internal Medicine, Yeouido St. Mary’s Hospital, The Catholic University of Korea College of Medicine, 10 63-ro, Yeongdeungpo-gu, Seoul 150-713, Korea

Tel: +82-2-3779-1519, Fax: +82-2-3779-1331, E-mail: adagio@catholic.ac.kr

${ }^{2}$ Department of Internal Medicine, Yeouido St. Mary's Hospital, The Catholic University of Korea College of Medicine, 10 63-ro, Yeongdeungpo-gu, Seoul 150-713, Korea

Tel: +82-2-3779-1079, Fax: +82-2-3779-1331, E-mail: jkkim@catholic.ac.kr

Received on December 8, 2013. Revised on April 10, 2014. Accepted on May 12, 2014. Published online December 5, 2014

pISSN 1976-2283 eISSN 2005-1212 http://dx.doi.org/10.5009/gnl13457

@ This is an Open Access article distributed under the terms of the Creative Commons Attribution Non-Commercial License (http://creativecommons.org/licenses/by-nc/4.0) which permits unrestricted non-commercial use, distribution, and reproduction in any medium, provided the original work is properly cited. 
a crucial role in tumor growth and glycolysis during tumorigenesis. ${ }^{6,7}$ The measurement of tumor M2-PK levels in the stool has been investigated to provide a new screening tool for CRC. ${ }^{8}$ A recent meta-analysis of fecal tumor M2-PK enzyme-linked immunosorbent assay (ELISA) test reported the sensitivity of $80.3 \%$ and the specificity of $95.2 \%$ for diagnosing CRC. ${ }^{9}$ The results are far more superior to previously reported values of FOBT. ${ }^{3}$ But, cost of ELISA is high and this test is not available in clinical practice. A new immunochromatographic qualitative method for fecal tumor M2-PK is developed. Similar to FOBT, this method is able to detect fecal tumor M2-PK by using preformed cassettes. But, the effectiveness of this method for detecting CRC and colonic adenoma has not yet been established.

We aimed to evaluate the usefulness of immunochromatographic M2 pyruvate kinase (iM2-PK) method for detecting CRC and colonic adenoma by comparing iFOBT, serum carcinoembryonic antigen (CEA) and fecal tumor M2-PK ELISA test.

\section{MATERIALS AND METHODS}

\section{Subjects and test modalities}

The value of iM2-PK was compared with iFOBT and fecal tumor M2-PK ELISA. The test was performed using fecal samples. Colonoscopy and histological result was used as gold standard.

From April 2012 to March 2013, healthy volunteers and patients with colorectal neoplasms were enrolled in six academic hospitals in capital province of Korea (Seoul St. Mary's Hospital, Uijeongbu St. Mary's Hospital, Bucheon St. Mary's Hospital, Incheon St. Mary's Hospital, St. Paul's Hospital, St. Vincent's Hospital of the Catholic University of Korea College of Medicine). A total of 323 subjects was recruited and consists of 60 healthy volunteers, 124 patients with colonic adenoma and 139 patients with CRC. The stool samples were collected from subjects awaiting surgery, out-patients and patients of health promotion clinics. All stool samples were collected before oral colonoscopy preparation administration. The iFOBT and iM2-PK were immediately performed with freshly collected stool samples. All 323 individuals underwent complete colonoscopy and blood sampling for CEA. The pathological tissue specimens were obtained by colonoscopic biopsies, polypectomies, or surgery. All those who had obvious gastrointestinal bleeding, menstruation, past history of colectomy, inflammatory bowel disease, and noncolonic gastrointestinal neoplasms were excluded.

Among 323 subjects, 68 were enrolled in ELISA M2-PK and iM2-PK comparison subgroup. The participants in this ELISA study consisted of 40 patients with colorectal cancer, 26 patients with adenoma and two healthy volunteers. Their stool samples were stored at $-20^{\circ} \mathrm{C}$ for ELSIA test. Fecal tumor M2-PK ELISA test was performed twice on each stool sample and the result data were reported as the mean value.

The informed consents were obtained from all participants before entering the study. Approval was obtained from the In- stitutional Review Boards (IRB) of the Catholic Medical Center of Korea prior to beginning this study (IRB number: XC12TIMI0035S).

\section{Methods}

\section{1) iFOBT}

The iFOBT strip device (Asan Easy Test FOB; Asan Biotech Institute, Seoul, Korea) was used. It provides an one-step immunochromatographic assay for rapid detection of fecal human blood. The stick of the sample collection device was put into the feces at three or four different sites and immediately inserted into the extraction buffer solution which was then shaken thoroughly. After that, two or three drops of this extracted sample were loaded into the sample opening of the test device. The liquid sample flows from the sample opening of the test device and conjugates to the gold labeled antihemoglobin antibodies binding to the human hemoglobin. These complexes migrate to the test membrane which has secondary antibodies binding to the gold-labeled antibody-hemoglobin complex and finally generated a violet band within 3 to 10 minutes.

\section{2) Immunochromatographic fecal tumor M2-PK test}

The mechanism of iM2-PK test is based on detecting tumor M2-PK by specific monoclonal antibodies. The process of the test has two stages. The tumor M2-PK reacts with a monoclonal antibody bound to gold particles and then, migrates along the membrane. And complex reaches the test line attached by a second monoclonal antibody. Gold-labeled antibody M2-PK complex binds to the test line and develops a pink color.

In this study, the stool samples were measured by using a commercially available cassette for iM2-PK (M2-PK Quick ${ }^{\mathrm{TM}}$; ScheBo ${ }^{\circledR}$ Biotech AG, Giessen, Germany). For sample preparation, the fresh stool specimen was extracted in the extraction buffer ( $\mathrm{pH}$ 7.2) mixed with 5\% detergent and 0.05\% sodium azide for 10 minutes. And then, four drops of the extracted sample were applied into the circular sample well on the cassette. The result was read after exactly 5 minutes. The negative result is that a pink band appears only in the control region and no band develops in the test lesion. And the positive result is that the pink band develops in both the control region and the test region. The test stripe must be clearly recognizable as a line, although it may be weaker than that of the control.

\section{3) Fecal tumor M2-PK ELISA test}

The fecal tumor M2-PK ELISA test was performed by using a commercially available kit (Tumor M2-PK ${ }^{\mathrm{TM}}$ ELISA Stool Test; ScheBo ${ }^{\circledR}$ Biotech AG). The ELISA kit is made as 96 well microtiter plates, suitable for up to 42 samples in duplicate. The wells of plate are coated with a monoclonal antibody against tumor M2-PK. The stool sample was extracted in extraction buffer and diluted in the washing buffer provided in the kit. The prepared samples were loaded on the ELISA plate and incubated for 60 
minutes at room temperature. The biotinylated second monoclonal antibodies in 1:00 dilution was added and incubated again for 30 minutes at the room temperature. A conjugate of peroxidase and streptavidin were added for binding to the biotin moiety, and incubated for another 30 minutes. Substrate solution and the stop solution were separately added and incubated for 15 minutes. Completed ELISA plate was read by photometry at OD $450 \mathrm{~nm}$ and the standard curve was calculated as the log$\log$ scale.

\section{Statistical analysis}

Statistical significance was determined by the Wilcoxon rank sum test and Kruskal-Wallis test. All statistical analyses were performed by using SAS software for Windows (release 9.3; SAS Institute, Cary, NC, USA) and p-values less than 0.05 were interpreted as statistically significant.

\section{RESULTS}

\section{Clinical characteristics}

Clinical characteristics including gender, social history, and underlying diseases with diabetes, hypertension, tuberculosis and hepatitis were not different between the groups except age. The control group included 60 healthy volunteers $(27$ males and 33 females; median age, 55 years; range, 40 to 79 years). The colonic adenoma group consisted of 124 patients (75 males and 49 females; median age, 63 years; range, 38 to 81 years). The 139 patients (82 males and 57 females; median age, 66 years; range, 36 to 92 years) were diagnosed with primary CRC. The clinical characteristics are shown in Table 1.

\section{Accuracy of the iM2-PK in terms of sensitivity, specificity, and predictive values}

The performance characteristics of iM2-PK, iFOBT, and serum CEA were evaluated for the study groups and the values are shown in Table 2. Sensitivity for CRC was significantly higher with iM2-PK (92.8\%) than iFOBT (47.5\%) and CEA (45.3\%) $(\mathrm{p}<0.05)$. Sensitivity for colorectal adenoma was significantly better with iM2-PK (69.4\%) than iFOBT (12.1\%) and CEA (46.8\%) $(\mathrm{p}<0.05)$. Specificity for CRC and adenoma was not different among tests.

Negative predictive value for CRC was significantly higher with iM2-PK (83.3\%) than iFOBT (40.7\%) and CEA (40.6\%) $(\mathrm{p}<0.05)$. For colorectal adenoma, negative predictive value was better with iM2-PK $(56.8 \%)$ than iFOBT $(31.5 \%)(p<0.05)$ but not than CEA (43.6\%). Positive predictive values for CRC and adenoma were not different among tests.

The receiver operating characteristic (ROC) curves of iM2PK, iFOBT, and CEA for predicting patients with adenoma and CRC are shown in Fig. 1. The curves are statistically significant with the areas beneath them which are greater than 50\%. The areas under the curves of iM2-PK are 0.88 for CRC and 0.76 for adenoma. These values are significantly higher than those of iFOBT and CEA $(\mathrm{p}<0.05)$. The threshold values of CEA, which were calculated as the most appropriate cutoff value using the ROC curve, were $1.28 \mathrm{U} / \mathrm{mL}$ for predicting adenoma and $3.14 \mathrm{U} /$ $\mathrm{mL}$ for predicting $\mathrm{CRC}$.

\section{Comparison iM2-PK with M2-PK ELISA test}

Comparison between iM2-PK, iFOBT, and M2-PK ELISA was possible in 68 subjects. The cutoff value of M2-PK ELISA test

Table 1. Clinical Characteristics of Three Different Study Groups

\begin{tabular}{|c|c|c|c|c|c|}
\hline Characteristic & $\begin{array}{c}\text { Total } \\
(n=323)\end{array}$ & $\begin{array}{c}\text { Normal } \\
(n=60)\end{array}$ & $\begin{array}{c}\text { Adenoma } \\
(\mathrm{n}=124)\end{array}$ & $\begin{array}{c}\text { CRC } \\
(n=139)\end{array}$ & $\mathrm{p}$-value \\
\hline \multicolumn{6}{|l|}{ Gender } \\
\hline Male & $184(57.0)$ & $27(45.0)$ & $75(60.5)$ & $82(59.0)$ & 0.113 \\
\hline Female & $139(43.0)$ & $33(55.0)$ & 49 (39.5) & $57(41.0)$ & \\
\hline Age & $62(36-92)$ & 55 (40-79) & $63(38-81)$ & 66 (36-92) & $<0.001$ \\
\hline Diabetes & $46(14.2)$ & $6(10.0)$ & $20(16.1)$ & $20(14.4)$ & 0.536 \\
\hline Hypertension & $117(36.2)$ & $15(25.0)$ & $59(47.6)$ & 43 (30.9) & 0.003 \\
\hline Tuberculosis & $15(4.6)$ & $6(10.0)$ & $3(2.4)$ & $6(4.3)$ & 0.070 \\
\hline Hepatitis & $13(4.0)$ & $5(8.3)$ & $3(2.4)$ & $5(3.6)$ & 0.178 \\
\hline Alcohol & 83 (25.7) & $10(16.7)$ & 40 (32.3) & 33 (23.7) & 0.060 \\
\hline \multicolumn{6}{|l|}{ Smoking } \\
\hline None & $250(77.4)$ & $52(86.7)$ & $93(75.0)$ & 105 (75.5) & 0.291 \\
\hline Former smoker & $40(12.4)$ & $4(6.7)$ & $15(12.1)$ & $21(15.1)$ & \\
\hline Current smoker & $33(10.2)$ & $4(6.7)$ & 16 (12.9) & $13(9.4)$ & \\
\hline
\end{tabular}

Data are presented as number (\%) or median (minimum-maximum).

$\mathrm{CRC}$, colorectal cancer. 
A

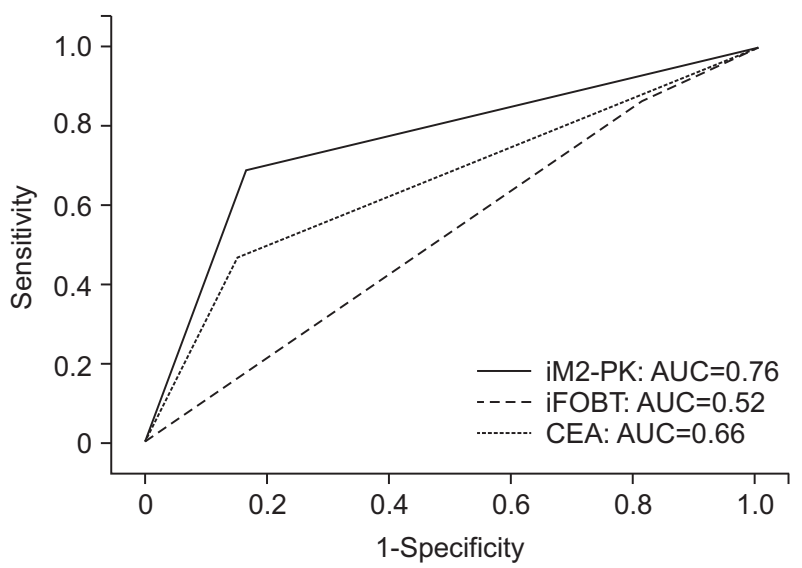

B

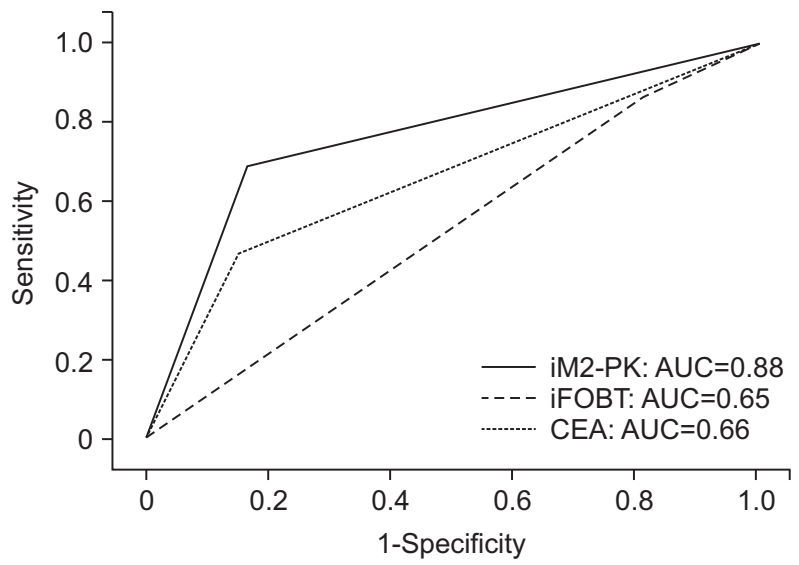

Fig. 1. The receiver-operating characteristic curves for predicting adenoma (A) and colorectal cancer (B). Each line present receiver-operating characteristic curve for predicting adenoma and colorectal cancer by immunochromatographic M2 pyruvate kinase, immunochromatographic fecal occult blood test and carcinoembryonic antigen. All the curves are statistically significnt $(\mathrm{p}<0.001)$, with the areas beneath the curves being greater than $50 \%$.

iM2-PK, immunochromatographic M2 pyruvate kinase; AUC, area under the curve; iFOBT, immnochromatographic fecal occult blood test; CEA, carcinoembryonic antigen.

Table 2. Sensitivity, Specificity, Predictive Values and Area under the Curves of the Tests Used for Detection of Colorectal Cancer and Adenoma

\begin{tabular}{|c|c|c|c|c|c|}
\hline Variable & iM2-PK & iFOBT & CEA & p-value $e^{*}$ & p-value ${ }^{\dagger}$ \\
\hline \multicolumn{6}{|l|}{ Sensitivity } \\
\hline CRC & 92.8 (87.1-96.5) & $47.5(38.9-56.1)$ & $45.3(36.8-53.9)$ & $<0.001$ & $<0.001$ \\
\hline Adenoma & $69.4(60.4-77.3)$ & $12.1(6.9-19.2)$ & $46.8(37.7-55.9)$ & $<0.001$ & $<0.001$ \\
\hline \multicolumn{6}{|l|}{ Specificity } \\
\hline CRC & 83.3 (71.4-91.1) & 83.3 (71.4-91.7) & 86.7 (75.4-94.0) & $>0.999$ & 0.609 \\
\hline Adenoma & 83.3 (71.4-91.7) & 83.3 (71.4-91.7) & 85.0 (73.4-92.9) & $>0.999$ & 0.803 \\
\hline \multicolumn{6}{|l|}{ PPV } \\
\hline CRC & $92.8(87.1-96.5)$ & 86.8 (77.1-93.5) & 88.7 (79.0-95.0) & 0.150 & 0.319 \\
\hline Adenoma & 89.6 (81.6-94.8) & $60.0(38.6-78.8)$ & 86.6 (76.0-93.6) & 0.001 & 0.555 \\
\hline \multicolumn{6}{|l|}{ NPV } \\
\hline CRC & 83.3 (71.4-91.7) & $40.7(31.8-49.8)$ & $40.6(32.0-49.6)$ & $<0.001$ & $<0.001$ \\
\hline Adenoma & $56.8(45.8-67.3)$ & $31.5(24.3-39.2)$ & $43.6(34.4-53.0)$ & $<0.001$ & 0.061 \\
\hline \multicolumn{6}{|l|}{ AUC } \\
\hline CRC & $0.88(0.83-0.93)$ & $0.65(0.59-0.72)$ & $0.66(0.60-0.72)$ & $<0.001$ & $<0.001$ \\
\hline Adenoma & $0.76(0.70-0.83)$ & $0.52(0.47-0.58)$ & $0.66(0.60-0.72)$ & $<0.001$ & 0.028 \\
\hline
\end{tabular}

Data are presented as percentage (95\% confidence interval).

iM2-PK, immunochromatographic M2 pyruvate kinase; iFOBT, immunochromatographic fecal occult blood test; CEA, carcinoembryonic antigen; CRC, colorectal cancer; PPV, positive predictive value; NPV, negative predictive value; AUC, area under the curves.

*Significant difference between iM2-PK and iFOBT based on Fisher exact test and chi-square test; ${ }^{\dagger}$ Significant difference between iM2-PK and CEA based on Fisher exact test and chi-square test.

was set as $4 \mathrm{U} / \mathrm{mL}$ according to results from a previous metaanalysis. ${ }^{9}$

The sensitivity and specificity of iM2-PK, iFOBT, and M2-PK ELISA test were calculated (Table 3). The sensitivity of iM2-PK for CRC was significantly better than M2-PK ELISA (97.5\% vs 80.0\%, p<0.05). But, sensitivity for adenoma and specificity for CRC and adenoma were not different between iM2-PK and M2PK ELISA.

\section{Sensitivity and specificity of iM2-PK according to the Dukes and TNM staging in colorectal cancer, the grade of dysplasia and the polyp size in colonic adenoma}

A total of 139 patients with CRC were classified into three groups according to Dukes staging: 31 patients with Dukes A, 31 patients with Dukes B, and 77 patients with Dukes C. The sensitivity of iM2-PK according to Dukes staging were 80.6\% in 
Table 3. The Sensitivity and Specificity of the Tests for Colorectal Cancer and Adenoma

\begin{tabular}{lccccc}
\hline \multicolumn{1}{c}{ Variable } & iM2-PK & iFOBT & M2-PK ELISA (>4 U/mL) & p-value $^{*}$ & $\mathrm{p}^{*}$ value $^{\dagger}$ \\
\hline Sensitivity & & & & & \\
CRC & $97.5(86.8-99.9)$ & $47.5(31.5-63.9)$ & $80.0(64.4-91.0)$ & $<0.001$ & 0.029 \\
Adenoma & $69.2(48.2-85.7)$ & $19.2(6.6-39.4)$ & $61.5(40.7-79.8)$ & $<0.001$ & 0.560 \\
Specificity & & & & $>0.999$ & $>0.999$ \\
CRC & $100.0(15.8-100.0)$ & $100.0(15.8-100.0)$ & $50.0(1.3-98.7)$ & $>0.999$ & $>0.999$ \\
Adenoma & $100.0(15.8-100.0)$ & $100.0(15.8-100.0)$ & $50.0(1.3-98.7)$ & & \\
\hline
\end{tabular}

Data are presented as percentage (95\% confidence interval).

iM2-PK, immunochromatographic M2 pyruvate kinase; iFOBT, immunochromatographic fecal occult blood test; ELISA, enzyme-linked immunosorbent assay; CRC, colorectal cancer.

*Significant difference between iM2-PK and iFOBT based on Fisher exact test and chi-square test; ${ }^{\dagger}$ Significant difference between iM2-PK and M2-PK ELISA based on Fisher exact test and chi-square test.

Table 4. Sensitivity and Specificity of iM2-PK Test according to the Grade of Dysplasia and the Size of the Polyp in Colonic Adenoma

\begin{tabular}{|c|c|c|c|c|c|c|}
\hline \multirow{2}{*}{ Variable } & \multicolumn{3}{|c|}{ Grade of dysplasia } & \multicolumn{3}{|c|}{ Size of polyp, mm } \\
\hline & LGD $(n=90)$ & HGD $(n=34)$ & p-value* & $<10(\mathrm{n}=30)$ & $\geq 10(\mathrm{n}=94)$ & p-value ${ }^{\dagger}$ \\
\hline \multicolumn{7}{|l|}{ iM2-PK } \\
\hline Positive & $56(62.2)$ & 30 (88.2) & & $19(63.3)$ & $67(71.3)$ & \\
\hline Negative & $34(37.8)$ & $4(11.8)$ & & $11(36.7)$ & 27 (28.7) & \\
\hline Sensitivity & $62.2(51.4-72.2)$ & 88.2 (72.6-96.7) & 0.005 & $63.3(43.9-80.1)$ & $71.3(61.0-80.1)$ & 0.411 \\
\hline
\end{tabular}

Data are presented as number (\%) or percentage (95\% confidence interval).

iM2-PK, immunochromatographic M2 pyruvate kinase; LGD, low grade dysplasia; HGD, high grade dysplasia.

*Significant difference between LGD and HGD based on Fisher exact test and chi-square test; ${ }^{\dagger}$ Significant difference between $<10$ and $\geq 10 \mathrm{~mm}$ based on Fisher exact test and chi-square test.

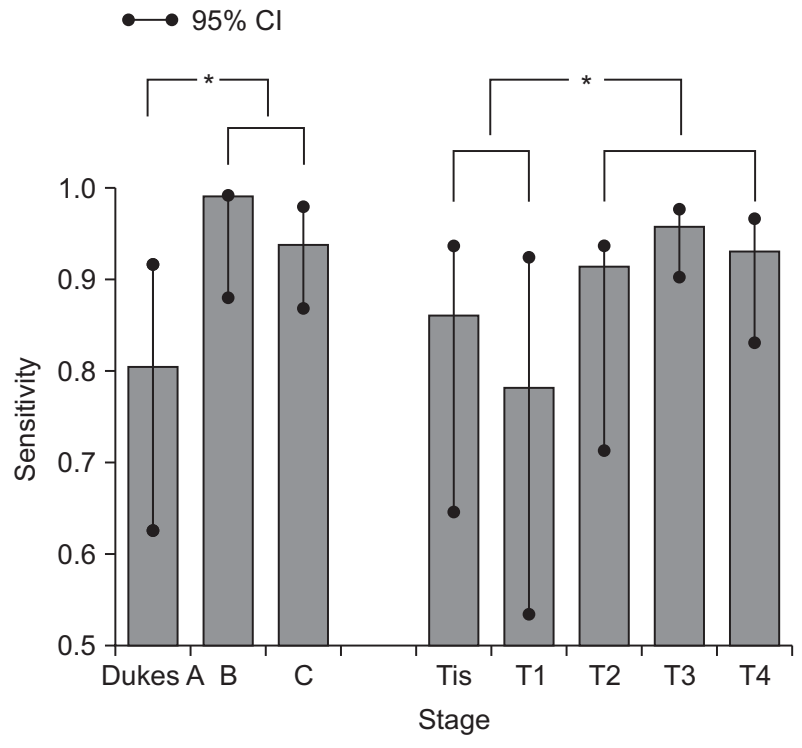

Fig. 2. The sensitivity of immunochromatographic M2 pyruvate kinase (iM2-PK) according to cancer stages based on Dukes and TNM classification. The sensitivity of iM2-PK of Dukes A stage cancer was 80.6\%. Cancers classified as Dukes B and Dukes C stages exhibited sensitivity of 100\% and 94.8\%, respectively. These values were significantly increased compared with cancers categorized as Dukes A stage $(\mathrm{p}<0.05)$. Similarly, cancers that were T2 or beyond exhibited significantly increased positivity compared with Tis and T1 cancers. The sensitivity for Tis and T1 cancers was $82.8 \%$, and the sensitivity for $\mathrm{T} 2, \mathrm{~T} 3$, and $\mathrm{T} 4$ cancers was $95.1 \%(\mathrm{p}=0.019)$.

CI, confidence interval. ${ }^{*} \mathrm{p}<0.05$.
Dukes A, 100.0\% in Dukes B, and 94.8\% in Dukes C. The sensitivities of Dukes B and Dukes C were significantly higher than that of Dukes A ( $p<0.05)$ (Fig. 2).

Regarding the TNM stages, nodal involvement did not influence on the sensitivity of iM2-PK test. The sensitivity of iM2-PK was 93.3\% for cancer with lymph node metastasis and 92.2\% for those without $(\mathrm{p}=0.794)$. The depth of cancer invasion affected the sensitivity of iM2-PK, but serial increment of the sensitivity did not exist when all the T stages, from Tis to T4, were compared ( $p=0.239$ ). The highest sensitivity was $96.8 \%$ for T3 cancers and the lowest was 78.6\% for T1 cancers. Only when cancers were grouped into those confined to mucosa and submucosa and others invading the muscle proper and beyond, the sensitivity of iM2-PK was higher in advanced cancer group than in early cancer group (95.1\% vs 82.8\%, $p=0.019$ ) (Fig. 2).

A total of 124 patients with colonic adenoma were classified into two groups according to the grade of dysplasia: 90 patients with low grade dysplasia and 34 patients with high grade dysplasia. The sensitivity of iM2-PK was $62.2 \%$ in low grade dysplasia, and $88.2 \%$ in high grade dysplasia. The sensitivity of HGD was significantly higher than that of low grade dysplasia $(\mathrm{p}=0.0051)$.

Among 124 patients with colonic adenoma, 30 patients had polyps less than $10 \mathrm{~mm}$ and 94 patients had a polyp over 10 $\mathrm{mm}$. The sensitivity was $63.3 \%$ in polyps less than $10 \mathrm{~mm}$, and 
71.3\% in a polyp over $10 \mathrm{~mm}$ (Table 4). There was no significant difference in sensitivity according to the size of the polyp $(\mathrm{p}=0.4113)$.

\section{DISCUSSION}

The pyruvate kinase (PK) is a key enzyme in glucose metabolism of glycolysis as dephosphorylation of phosphoenolpyruvate to pyruvate. There are several types of tissue specific PK. L-PK is found in the liver, M1-PK in muscle and brain, R-PK in erythrocytes, and M2-PK in undifferentiated and proliferating tissues. In gastrointestinal tract, M2-PK is the only detectable PK and exists mainly as a dimeric form. The M2-PK initially formed the active tetramer and then it split into stable dimeric forms. The stable dimeric form of M2-PK is usually called as a tumor M2-PK. ${ }^{10}$ In cancer cells, M2-PK regulates the synthetic balance between ATP and macromolecules. The aim of this study was to examine whether iM2-PK is useful in screening for CRC and colonic adenoma.

The most common screening method for diagnosing CRC is FOBT which detects hemoglobin in stool samples enzymatically or immunologically. ${ }^{11}$ The old enzymatic FOBT which is referred as guaiac FOBT (gFOBT) detects the peroxidase activity of hemoglobin. The gFOBT can be affected by dietary peroxidases contained in fruits or vegetables, dietary hemes from red meat which causes false positive result, and antioxidants such as vitamin $C$ which may cause false negative result. ${ }^{12}$ The sensitivity of gFOBT is relatively low and varied in many studies, between 30 and $80 \% .{ }^{13-15}$ However, the newer iFOBT uses specific antibodies binding to the human hemoglobin. iFOBT results can be less affected by diet or some medicine and achieve better outcomes. iFOBT is recognized a useful screening test in many countries running population based CRC screening program. ${ }^{16}$ Nonetheless, other causes of gastrointestinal bleeding such as nonsteroidal anti-inflammatory drugs injury and benign ulcers, could cause false positive results. ${ }^{17}$ According to a previous analysis, the sensitivity of iFOBT was superior to that of gFOBT as well as the specificity and positive predictive values in cohort trials of the surveillance patients diagnosed for advanced colorectal neoplasm. ${ }^{3}$ However, FOBTs are specific to hemoglobin but not to specific to colorectal neoplasm. To find the reason for positive results of FOBTs, one should undergo colonoscopy. ${ }^{18}$

Previous studies investigating the fecal tumor M2-PK ELISA test reported that the sensitivity for CRC ranges from about 70\% to $80 \%$ and the specificity ranges from about $70 \%$ to $90 \%{ }^{19-25}$ The tumor M2-PK ELISA test is more specific to CRC than iFOBT. ${ }^{26}$ The weak point of M2-PK lies on the fact that it is sensitive to intestinal inflammation in patients with different colorectal diseases such as inflammatory bowel disease, diarrhea, diverticulitis, and pouchitis. ${ }^{27}$ However, for diagnosing colonic adenoma, the sensitivity of $22 \%$ and the specificity of $82 \%$ were reported in a large-scale study from Germany and the result had a very limited potential for detecting adenomas. ${ }^{28}$

The fecal tumor M2-PK ELISA test has some problems to be used in clinical practice. Fecal tumor M2-PK ELISA test is timeconsuming. It usually takes around 6 hours to produce the reliable results. Meanwhile, only 10 minutes is necessary to go through this process with the office-based iFOBT. ${ }^{29}$ Moreover, if there is no available laboratory in a hospital, the M2-PK ELISA test may require additional time because the stool samples should be sent to the available laboratory and must be stored in a frozen state under $-20^{\circ} \mathrm{C}$. High cost is another obstacle. One of the most important considerations for introducing a new screening test is cost-effectiveness. The cost of the fecal tumor M2-PK ELISA test is about five times more expensive than that of iFOBT. ${ }^{29}$

iM2-PK is an immunochromatographic qualitative method for fecal tumor M2-PK. This test is performed in a short time (about 15 minutes) and the cost is about half of the fecal tumor M2-PK ELISA test. The main advantage of the iM2-PK is that it is quickly performed with fresh stool samples in clinics without being sent to a laboratory. In this study of the iM2-PK, the sensitivity was superior to that of the fecal tumor M2-PK ELISA test reported in previous studies ranged from about $70 \%$ to $80 \%$ and the specificity was within the range of previously reported values ranged from about 70\% to 90\%. ${ }^{19-25}$ Moreover, the ROC curves of this study indicate that the iM2-PK may have a better diagnostic accuracy than those of iFOBT and serum CEA for diagnosing CRC as well as colonic adenoma (Table 2).

The sensitivity of iM2-PK for adenoma (69.4\%) was higher than that of iFOBT (12.1\%) in this study. The ELISA study was carried out in a single center with 68 subjects for the purpose of comparing with the iM2-PK and the fecal tumor M2-PK ELISA test. The sensitivities of iM2-PK were higher than those of the fecal tumor M2-PK ELISA test in diagnosing CRC (Table 3). The reason for the low sensitivity of ELISA test might be found from the bio-stability of tumor M2-PK in stool sample. Long-time preservation and handling time might induce degradation of tumor M2-PK proteins. In a previous report about the stability of tumor M2-PK, the sensitivity of fecal tumor M2-PK ELISA test were decreased gradually from $67.8 \%$ to $36.9 \%$ according to the days of storage at room temperature. ${ }^{30}$

Regarding the power of screening tests, sensitivity and specificity have the power in opposite directions. In this study results, the sensitivity of iM2-PK is about two times high compared to that of iFOBT, but the specificity was $83 \%$ which is lower than the usually reported value of iFOBT in other literatures. This aspect may evoke a concern that unnecessary investigations including colonoscopy will be taken in patients with false positive result with iM2-PK. In population-based or government-directed mass screening, specificity is a more important factor to reduce the medical budget with limited resource. On the contrary, for the patient, the sensitivity is considered more valued in order not to miss presence of cancer or premalignant lesions. In this 
study, iM2-PK test proved to have a modest power in specificity and a far advanced power in sensitivity, compared to iFOBT. In these days, the availability and safety of colonoscopy is markedly expanded in Korea as well as other developed nations. The financial affordability of individual and government also has grown to pursue better quality of life, not only just saving of life. It is possible that iM2-PK may result in unnecessary investigations in some individuals. However, this test can provide better chances for early detection and successful treatment of colorectal cancer in others.

In this study, we classified CRC with Dukes and TNM staging. There were no patients with Dukes D because only resectable patients were included in this study. The sensitivities of the iM2PK in Dukes B and Dukes C were significantly higher than the sensitivity in Dukes A. And there was no significant difference between Dukes B and Dukes C (Fig. 2). Similarly the sensitivity of the iM2-PK was not different according to T stages and $\mathrm{N}$ stages ( $p=0.239$ and $p=0.794)$. Only when authors grouped cancers confined to mucosa and submucosa and cancers invading the muscle proper and beyond, the sensitivity of iM2-PK was higher in advanced cancer group (95.1\%) than in early cancer group $(82.8 \%)(\mathrm{p}=0.019)$. This means that the fecal tumor M2PK may be more detectable in the advanced stage rather than the early stage of CRC. This could be explained that the detection of fecal tumor M2-PK might be dependent on intraluminal volume of cancer which may or may not have lymph node metastasis. We also classified colonic adenomas according to the grade of dysplasia and the size of polyp. The fecal tumor M2-PK was more detectable in high grade dysplasia than in low grade dysplasia and there was no significant difference according to the size of polyp in this study. It can be assumed that a larger number of adenomas may make the relationship between the iM2-PK sensitivity and size statistically significant.

In conclusion, iM2-PK test proved to have significantly higher sensitivity for CRC and colon adenomatous lesions than that of iFOBT and CEA. The negative predictive value of iM2-PK was also better than iFOBT. The iM2-PK has strength in its affordable cost, convenience in use and the rapidity for result. iM2-PK may be a reliable and acceptable screening method for CRC.

\section{CONFLICTS OF INTEREST}

No potential conflict of interest relevant to this article was reported.

\section{ACKNOWLEDGEMENTS}

Authors appreciate that this study was supported financially by Alere Healthcare Inc., Seongnam, Korea.

The statistical consultation was supported by Catholic Research Coordinating Center of the Korea Health 21 R\&D Project
(A070001), Ministry of Health and Welfare, Korea.

\section{REFERENCES}

1. Jung KW, Won YJ, Kong HJ, Oh CM, Seo HG, Lee JS. Cancer statistics in Korea: incidence, mortality, survival and prevalence in 2010. Cancer Res Treat 2013;45:1-14.

2. Jemal A, Bray F, Center MM, Ferlay J, Ward E, Forman D. Global cancer statistics. CA Cancer J Clin 2011;61:69-90.

3. Zhu MM, Xu XT, Nie F, Tong JL, Xiao SD, Ran ZH. Comparison of immunochemical and guaiac-based fecal occult blood test in screening and surveillance for advanced colorectal neoplasms: a meta-analysis. J Dig Dis 2010;11:148-160.

4. Tagore KS, Lawson MJ, Yucaitis JA, et al. Sensitivity and specificity of a stool DNA multitarget assay panel for the detection of advanced colorectal neoplasia. Clin Colorectal Cancer 2003;3:4753.

5. Ahlquist DA, Skoletsky JE, Boynton KA, et al. Colorectal cancer screening by detection of altered human DNA in stool: feasibility of a multitarget assay panel. Gastroenterology 2000;119:1219-27.

6. Christofk HR, Vander Heiden MG, Wu N, Asara JM, Cantley LC. Pyruvate kinase M2 is a phosphotyrosine-binding protein. Nature 2008;452:181-186.

7. Christofk HR, Vander Heiden MG, Harris MH, et al. The M2 splice isoform of pyruvate kinase is important for cancer metabolism and tumour growth. Nature 2008;452:230-233.

8. Hardt PD, Ngoumou BK, Rupp J, Schnell-Kretschmer H, Kloer HU. Tumor M2-pyruvate kinase: a promising tumor marker in the diagnosis of gastro-intestinal cancer. Anticancer Res 2000;20:49654968.

9. Tonus C, Sellinger M, Koss K, Neupert G. Faecal pyruvate kinase isoenzyme type M2 for colorectal cancer screening: a meta-analysis. World J Gastroenterol 2012;18:4004-4011.

10. Mazurek S, Grimm H, Oehmke M, Weisse G, Teigelkamp S, Eigenbrodt E. Tumor M2-PK and glutaminolytic enzymes in the metabolic shift of tumor cells. Anticancer Res 2000;20:5151-5154.

11. Huang CS, Lal SK, Farraye FA. Colorectal cancer screening in average risk individuals. Cancer Causes Control 2005;16:171-188.

12. Feinberg EJ, Steinberg WM, Banks BL, Henry JP. How long to abstain from eating red meat before fecal occult blood tests. Ann Intern Med 1990;113:403-404.

13. Hewitson P, Glasziou P, Watson E, Towler B, Irwig L. Cochrane systematic review of colorectal cancer screening using the fecal occult blood test (hemoccult): an update. Am J Gastroenterol 2008;103:1541-1549.

14. Malila N, Oivanen T, Malminiemi 0, Hakama M. Test, episode, and programme sensitivities of screening for colorectal cancer as a public health policy in Finland: experimental design. BMJ 2008;337:a2261.

15. Whitlock EP, Lin JS, Liles E, Beil TL, Fu R. Screening for colorectal cancer: a targeted, updated systematic review for the U.S. Preven- 
tive Services Task Force. Ann Intern Med 2008;149:638-658.

16. Allison JE, Fraser CG, Halloran SP, Young GP. Population screening for colorectal cancer means getting FIT: the past, present, and future of colorectal cancer screening using the fecal immunochemical test for hemoglobin (FIT). Gut Liver 2014;8:117-130.

17. Lynch NM, McHutchison JG, Young GP, Deacon M, St John DJ, Barraclough D. Gastrointestinal blood loss from a new buffered aspirin (Ostoprin): measurement by radiochromium and Hemoquant techniques. Aust N Z J Med 1989;19:89-96.

18. Duffy MJ, van Rossum LG, van Turenhout ST, et al. Use of faecal markers in screening for colorectal neoplasia: a European group on tumor markers position paper. Int J Cancer 2011;128:3-11.

19. Hardt PD, Mazurek S, Toepler M, et al. Faecal tumour M2 pyruvate kinase: a new, sensitive screening tool for colorectal cancer. Br J Cancer 2004;91:980-984.

20. Vogel T, Driemel C, Hauser A, et al. Comparison of different stool tests for the detection of cancer of the colon. Dtsch Med Wochenschr 2005; 130:872-877.

21. Shastri YM, Naumann M, Oremek GM, et al. Prospective multicenter evaluation of fecal tumor pyruvate kinase type M2 (M2PK) as a screening biomarker for colorectal neoplasia. Int J Cancer 2006;119:2651-2656.

22. Tonus C, Neupert G, Sellinger M. Colorectal cancer screening by non-invasive metabolic biomarker fecal tumor M2-PK. World J Gastroenterol 2006;12:7007-7011.

23. Haug U, Rothenbacher D, Wente MN, Seiler CM, Stegmaier C, Brenner H. Tumour M2-PK as a stool marker for colorectal cancer: comparative analysis in a large sample of unselected older adults vs colorectal cancer patients. Br J Cancer 2007;96:1329-1334.

24. Mulder SA, van Leerdam ME, van Vuuren AJ, et al. Tumor pyruvate kinase isoenzyme type M2 and immunochemical fecal occult blood test: performance in screening for colorectal cancer. Eur J Gastroenterol Hepatol 2007;19:878-882.

25. Koss K, Maxton D, Jankowski JA. Faecal dimeric M2 pyruvate kinase in colorectal cancer and polyps correlates with tumour staging and surgical intervention. Colorectal Dis 2008;10:244-248.

26. Abdullah M, Rani AA, Simadibrata M, Fauzi A, Syam AF. The value of fecal tumor M2 pyruvate kinase as a diagnostic tool for colorectal cancer screening. Acta Med Indones 2012;44:94-99.

27. Walkowiak J, Banasiewicz T, Krokowicz P, Hansdorfer-Korzon R, Drews M, Herzig KH. Fecal pyruvate kinase (M2-PK): a new predictor for inflammation and severity of pouchitis. Scand J Gastroenterol 2005;40:1493-1494.

28. Haug U, Hundt S, Brenner H. Sensitivity and specificity of faecal tumour M2 pyruvate kinase for detection of colorectal adenomas in a large screening study. Br J Cancer 2008;99:133-135.

29. Shastri YM, Loitsch S, Hoepffner N, et al. Comparison of an established simple office-based immunological FOBT with fecal tumor pyruvate kinase type M2 (M2-PK) for colorectal cancer screening: prospective multicenter study. Am J Gastroenterol 2008;103:14961504.

30. Haug U, Wente MN, Seiler CM, Rothenbacher D, Büchler MW, Brenner H. Tumor M2 pyruvate kinase as a stool marker for colorectal cancer: stability at room temperature and implications for application in the screening setting. Clin Chem 2006;52:782784. 